\title{
A Compact Bandwidth Enhanced Antenna Loaded with SRR For WLAN/WiMAX/Satellite Applications
}

\author{
Tanweer Ali ${ }^{1 *}$, Mohammad Saadh AW ${ }^{2}$, Rajashekhar C. Biradar ${ }^{3}$ \\ ${ }^{1}$ Department of E\&C, Manipal Institute of Technology, Manipal Academy of Higher Education, Manipal, India \\ ${ }^{2,3}$ School of ECE, REVA University, Bangalore, India \\ *corresponding author, E-mail: tanweer.ali@manipal.edu
}

\begin{abstract}
A compact bandwidth enhanced antenna using Split Ring Resonator (SRR) for WLAN/WiMAX/Satellite applications is presented. As the wireless modules continue to shrink, there exists a drastic demand for a compact antenna. In addition to this, the effective integration of an antenna in wireless devices acts as stand-alone implement to mitigate the effects of noise fading. The presented antenna consists of a split ring resonator, two $\mathrm{C}$-shaped slot and a coaxial feed. Compactness in the design is achieved with the help of coaxial feeding technique. The etching of SRR creates a resonance at $6 \mathrm{GHz}$ (WLAN) band, with $\mathrm{S}_{11}<-10 \mathrm{~dB}$ bandwidth ranging from $5.46-6.7 \mathrm{GHz}(1240 \mathrm{MHz})$. Bandwidth enhancement in the proposed design (at $6 \mathrm{GHz}$ ), is accomplished by the two $\mathrm{C}$-shaped slots placed at the left and ride side of the radiating patch, just below the SRR. Due to the placement of two C-shaped slots the bandwidth of the proposed antenna gets enhanced from 1240 to 3120 MHz. The antenna has a size of only $0.4 \lambda_{0} \times 0.36 \lambda_{0} \times$ $0.05 \lambda_{0}=20 \times 18 \times 2.54$, at a resonance frequency of 6 GHz. The antenna exhibits bi-directional and Omnidirectional radiation characteristics in $\mathrm{E}$ and H-plane, respectively. Compact size, good impedance matching and stable radiation patterns are observed across the operational bandwidths. The aforementioned advantages make the proposed antenna suitable for WLAN/WiMAX/Satellite applications.
\end{abstract}

\section{Introduction}

A microstrip patch antenna has the advantage of light weight, compact physical dimensions, low cost and ease of installation, due to which they are used in high performance spacecraft, missiles, satellite and wireless handheld devices [1]. Despite, numerous advantages these antennas have the limitations of gain, bandwidth and efficiency [2]. In recent years, metamaterial have drawn great interest due to its favoring attributes like multiband operation, miniaturization, high data transmission rate and enhance bandwidth [3-4]. The Split Ring Resonator (SRR) [5-6] and Complementary Split Ring Resonator (CSRR) are extensively used structures in designing of a compact metamaterial microstrip antenna. The electrodynamics of the above mentioned metamaterial structures was first explained by Veselago [7]. The demand for wide bandwidth antenna with high data rate is increasing every year. Wideband antennas have the advantage of less interference, mitigates fading effect, have high data rates, less complex, low cost and resistant to multipath and jamming [8-9].

In this study, a compact bandwidth enhanced coaxial fed antenna loaded with SRR and slots is presented. The SRR in the radiating patch is used to obtain a resonance at $6 \mathrm{GHz}$ (WLAN) with a bandwidth of $1240.2 \mathrm{MHz}$, and the two Cshaped slots placed below it on the right and left side of the radiating patch, are used to increase the bandwidth of the antenna. The SRR is analyzed in a waveguide medium to achieve necessary S-parameters. The design structure is simulated using HFSS v.13.0 on RT Duroid 6006 substrate with a dielectric constant of 6.15 , height of $2.54 \mathrm{~mm}$, and loss tangent of 0.0019 .

\subsection{Problem Statement}

The tremendous growth in wireless communication has enabled the designers to create a plethora of smart applications, wearables and trackers. The growth of wireless communication is effected by devices with weaker signals, noise fading and limited battery life. However, careful antenna integration assures efficient performance of these wireless devices.

\subsection{Objective}

The main objective of this research is to design a bandwidth enhanced antenna which can be used for WLAN/WiMAX/Satellite applications by mitigating the effects of the aforementioned issues.

\subsection{Contributions}

In this study, a compact bandwidth enhanced coaxial fed antenna loaded with SRR is presented. The main contributions are as follows:-

- Bandwidth enhancement from $1240 \mathrm{MHz}$ to $3120 \mathrm{MHz}$ as compared to the conventional antenna.

- Stable omnidirectional radiation pattern in H-plane and Bidirectional radiation pattern in E-plane.

- Radiation efficiency greater than $95 \%$ across the entire operational bandwidths. 


\section{Design Evolution of Antenna}

The evolution stages of the proposed design are illustrated in Figure 1. As the design is completely based on the placement of SRR, the configuration "\#1" of Figure 1 is just a platform to place the SRR. In this stage (i.e. “\#1") a cylindrical stub is etched out in the radiating patch and the entire configuration is fed with coaxial feeding technique. The simulated result of this stage is illustrated in Figure 2. It can be observed that no operating bands are exbited by this antenna.

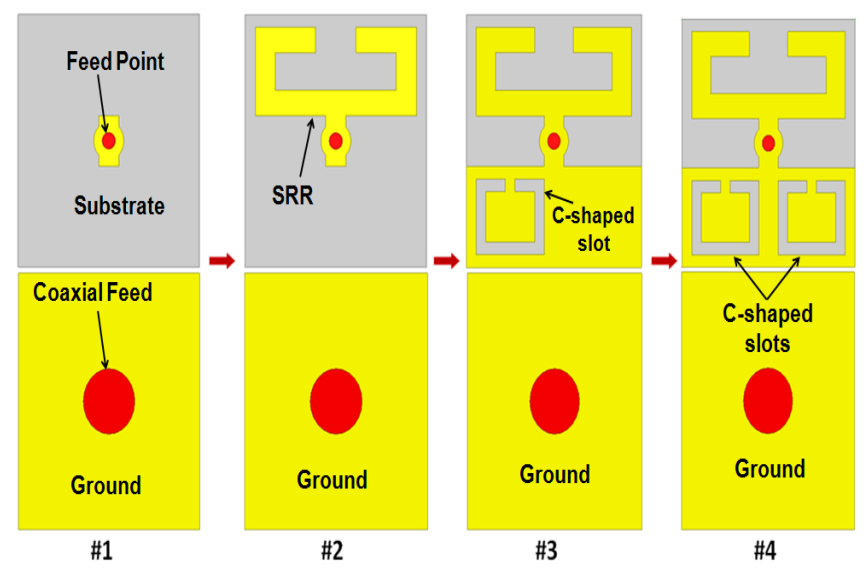

Figure 1. Proposed antenna design evolution

In configuration "\#2" of Figure 1, a SRR is placed on the top of cylindrical stub (i.e. upper part of radiating patch) to obtain a resonance at $6 \mathrm{GHz}$, as seen in Figure 2. The pass band phenomenon at $6 \mathrm{GHz}$ of this SRR is studied using waveguide medium and is discussed in detail in section 3.2. The introduction of this SRR makes the antenna to operate at $6 \mathrm{GHz}$ (WLAN) with $S_{11}<-10 \mathrm{~dB}$ bandwidth of about $1240 \mathrm{MHz}(5.46-6.7 \mathrm{GHz})$. In order to further improve the bandwidth, a C-shaped slot is etched out in the lower left section of "\#2" as seen in configuration "\#3" of Figure 1. Due to the introduction of this slot, surface current distribution of the antenna is further affected which tends the total current length path to increase as a result of which the antenna exhibits wider bandwidth from $4.65-7.95 \mathrm{GHz}$ (3300 MHz). However, the achieved bandwidth is wide but the impedance matching is not good (Figure 2, configuration "\#3"). In order to further match the impedance another Cshaped slot is etched just beside the first C-shaped slot, as depicted in configuration "\#4" of Figure 1. Due to the introduction of this symmetrical C-shaped slot the desired impedance matching is achieved, but as a consequence of this matching the bandwidth gets little narrower from 4.857.97 $\mathrm{MHz}(3120 \mathrm{MHz})$. Nevertheless, the achieved bandwidth is wider as compared to configuration "\#2". Thus, the proposed configuration exhibits the bandwidth enhancement from $1240 \mathrm{MHz}$ to $3120 \mathrm{MHz}$ with good impedance matching over the operational bandwidths, thereby easily meeting the criteria for WLAN/WiMAX/Satellite applications.

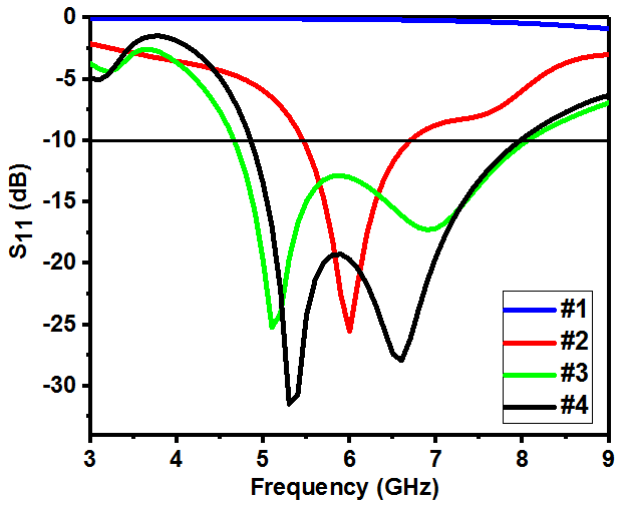

Figure 2. Design evolution reflection coefficient $\left(\mathrm{S}_{11}\right)$

The detailed layout dimension of the proposed configuration is demonstrated in Figure 3. It can be observed that the antenna consists of a SRR, two C-shaped slots and a coaxial feed. SRR $\left(\mathrm{S}_{3} \times \mathrm{S}_{2}\right)$ is placed at the upper part of the radiating patch and is the fundamental SRR to produce operating band at $6 \mathrm{GHz}, \mathrm{C}$-shaped slots $\left(\mathrm{S}_{6} \times \mathrm{S}_{9}\right)$ and $\left(\mathrm{S}_{7} \times\right.$ $\mathrm{S}_{10}$ ) are placed at the left and right side of the radiating patch just below the fundamental SRR to obtain bandwidth enhancement at $6 \mathrm{GHz}$. Coaxial feeding technique is used to achieve an impedance matching of $50 \mathrm{ohms}$.

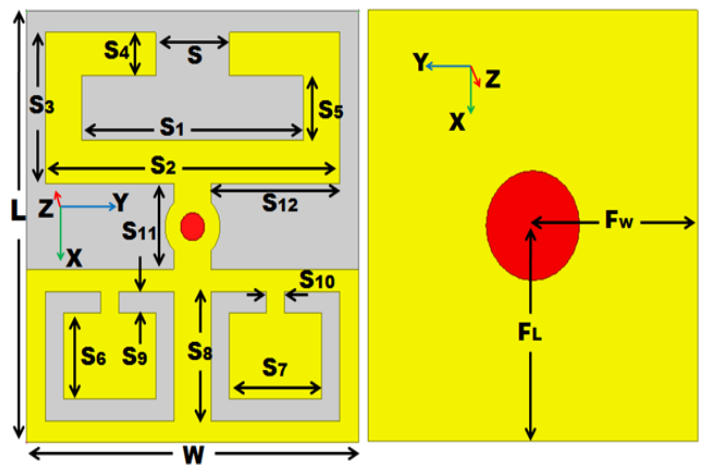

Figure 3. Detailed layout of the proposed design

$\mathrm{L}=20 \mathrm{~mm}, \mathrm{~W}=18 \mathrm{~mm}, \mathrm{~F}_{\mathrm{L}}=10 \mathrm{~mm}, \mathrm{~F}_{\mathrm{W}}=9 \mathrm{~mm}, \mathrm{~S}=4$ $\mathrm{mm}, \mathrm{S}_{1}=12 \mathrm{~mm}, \mathrm{~S}_{2}=16 \mathrm{~mm}, \mathrm{~S}_{3}=7 \mathrm{~mm}, \mathrm{~S}_{4}=2 \mathrm{~mm}, \mathrm{~S}_{5}=$ $3 \mathrm{~mm}, \mathrm{~S}_{6}=4 \mathrm{~mm}, \mathrm{~S}_{7}=5 \mathrm{~mm}, \mathrm{~S}_{8}=6 \mathrm{~mm}, \mathrm{~S}_{9}=1 \mathrm{~mm}, \mathrm{~S}_{10}=$ $1 \mathrm{~mm}, \mathrm{~S}_{11}=4 \mathrm{~mm}, \mathrm{~S}_{12}=7 \mathrm{~mm}$.

\section{Analysis of the Proposed Antenna}

\subsection{Design Equation}

The conventional design is calculated as in [1], where $\mathrm{W}$ and $\mathrm{L}$ are width and length of the patch respectively.

$$
\begin{aligned}
& W=\frac{c}{2 f_{r}} \sqrt{\frac{2}{\varepsilon_{r}+1}} \\
& L=\frac{c}{2 f_{r} \sqrt{\varepsilon_{\text {reff }}}}-2 \Delta L
\end{aligned}
$$


Where 'c' is the velocity of light $\left(3 \times 10^{8} \mathrm{~m} / \mathrm{s}\right)$, ' $f_{r}$ ' is the resonance frequency $(6 \mathrm{GHz})$, ' $\varepsilon_{r}$ ' is dielectric constant (6.15) of substrate used, ' $\varepsilon_{\text {reff }}$ ' is effective dielectric constant and ' $\Delta L$ ' is extension length. The width and length of the ground plane is calculated as in [1].

$$
\begin{gathered}
W_{g}=6 h+W \\
L_{g}=6 h+L
\end{gathered}
$$

\subsection{Analysis of Split Ring Resonator (SRR)}

The SRR of the proposed antenna structure is placed inside a waveguide medium and its $\mathrm{S}$ - parameters are retrieved as demonstrated in Figure 4, and the entire analysis is based on [10-15]. The upper and lower boundary of waveguide medium is assigned with PEC, front and back boundary is assigned with PMC and wave port excitation are applied on the either sides of the waveguide medium (Port 1 and Port 2). From the real and imaginary values of S-parameters, obtained from the waveguide set-up, the viable medium parameters like permittivity $(\varepsilon)$, and refractive index (n) are calculated and are illustrated in Figure 5. The calculations of these parameters are based on [9-14].

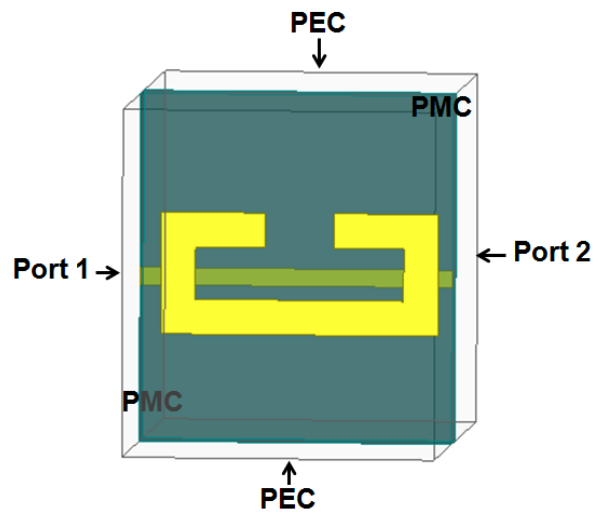

(a)

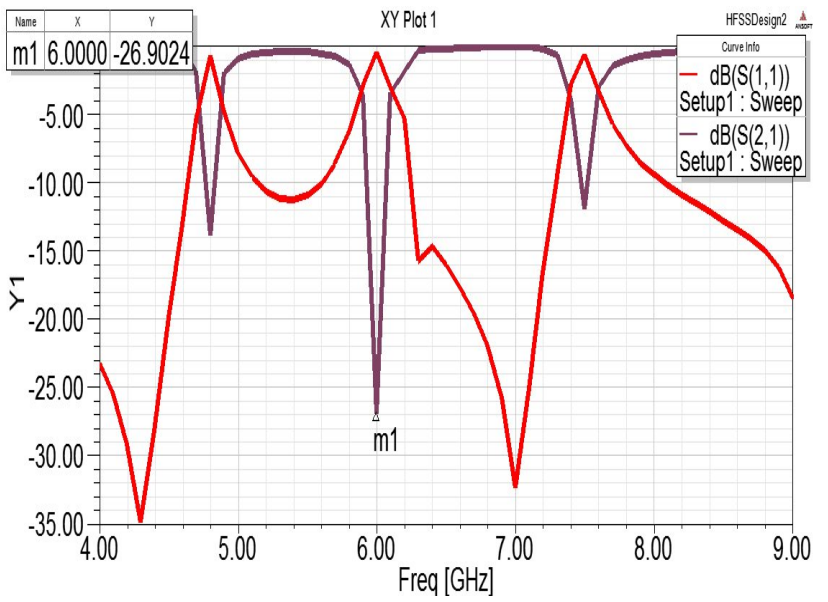

(b)

Figure 4. (a). Wave guide setup to retrieve $\mathrm{S}$ - parameters (b)retrieved $\mathrm{S}$ - parameters
From Figure 5(a), it can be observed that the negative permittivity (real) is observed at $6 \mathrm{GHz}$, which proves that this band exists due to the pass band phenomenon of the proposed single SRR.

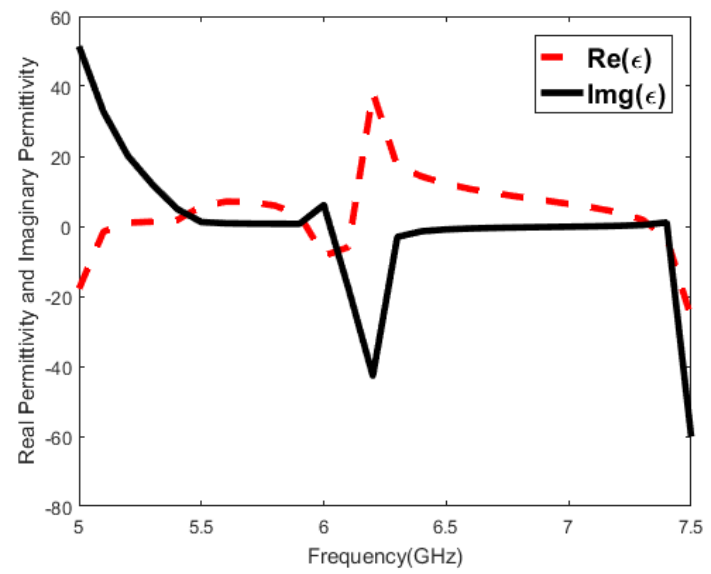

(a)

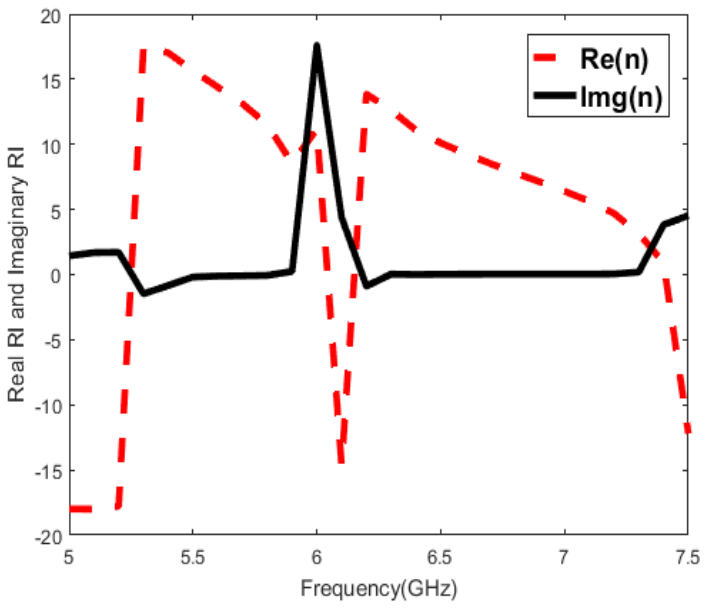

(b)

Figure 5. Retrieved (a) permittivity (b) refractive index

The examined SRR is placed on the upper section of configuration "\#1" to evolve into configuration "\#2", as illustrated in Figure 1. The reflection coefficient $\left(\mathrm{S}_{11}\right)$ of this structure ("\#2") is illustrated in Figure 6.

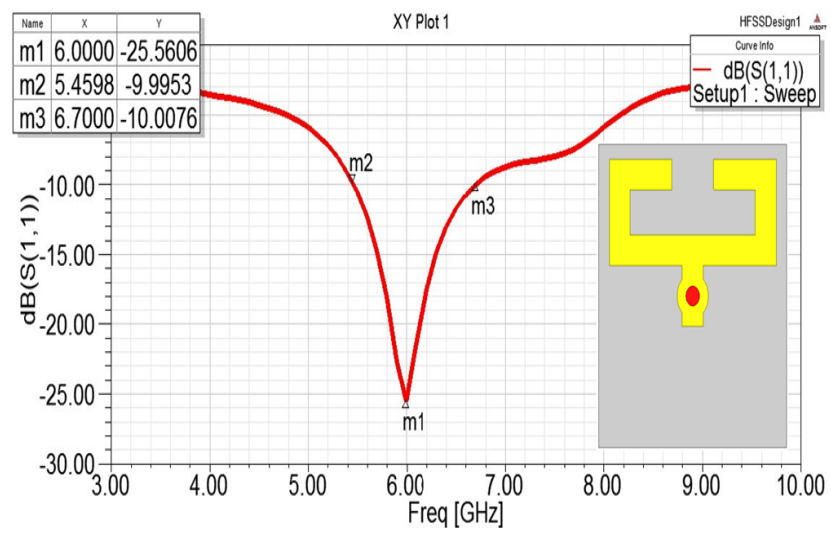

Figure 6 . Reflection coefficient $\left(\mathrm{S}_{11}\right)$ of configuration “\#2 


\subsection{Parametric Analysis}

To analyze the performance of the proposed design, parametric studies are done and are illustrated in Fig. 7. In the first case of study, the split gap (S) of the SRR structure is varied from 2 to $6 \mathrm{~mm}$ at a step of $2 \mathrm{~mm}$. The obtained $\mathrm{S}_{11}$ graph corresponding to this variation is illustrated in Figure 7. It can be observed that the best results are achieved with $\mathrm{S}$ $=4 \mathrm{~mm}$.

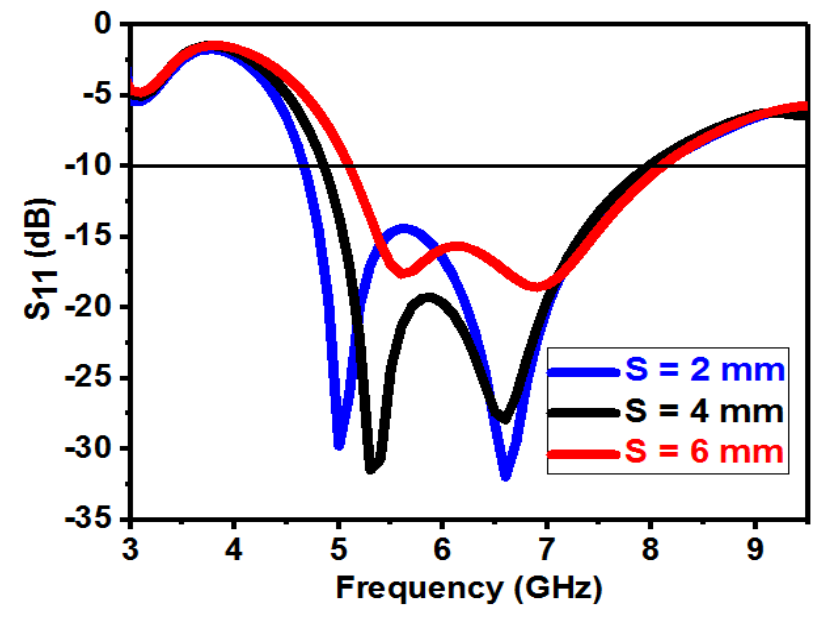

(a)

In the second case, to study the impedance matching phenomenon of the proposed design, the width $\left(\mathrm{S}_{4}\right)$ of the proposed SRR is varied from 1 to $3 \mathrm{~mm}$, at a step of $1 \mathrm{~mm}$. The obtained $S_{11}$ graph corresponding to this variation is illustrated in Figure 8. It can be seen that at $\mathrm{S}_{4}=1$ and $3 \mathrm{~mm}$, impedance and bandwidth of the proposed design gets affected as compared to $\mathrm{S}_{4}=2 \mathrm{~mm}$.

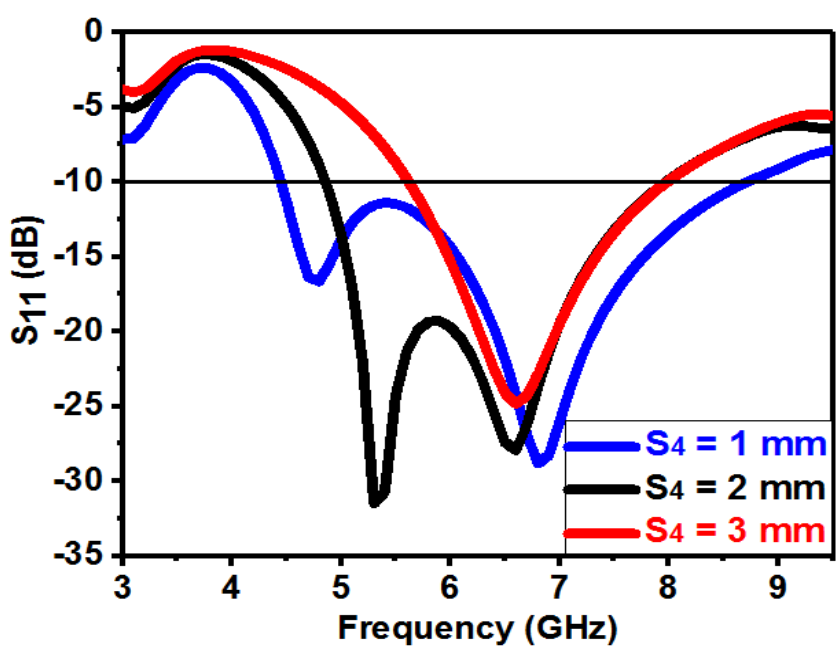

(b)

Similarly for third and fourth case, the effect of C-shaped slots $\left(\mathrm{S}_{9}\right)$ and $\left(\mathrm{S}_{10}\right)$ on the bandwidth of the proposed design are studied. It can observed from Figure 7 (c) and (d) that the best enhanced bandwidth is achieved with $\mathrm{S}_{9}=1 \mathrm{~mm}$ and $\mathrm{S}_{10}=1 \mathrm{~mm}$.

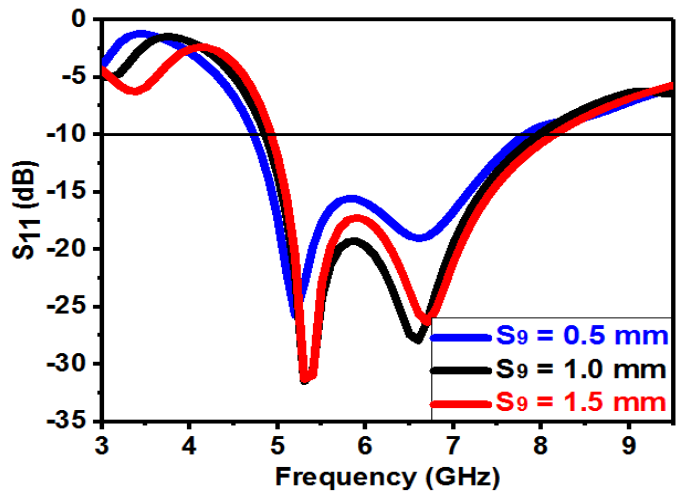

(c)

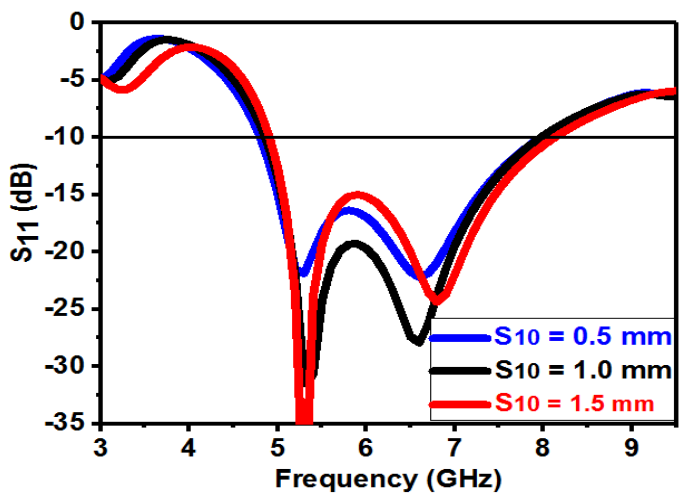

(d)

Figure 7. Parametric study for (a) $\mathrm{S}$, (b) $\mathrm{S}_{4}$, (c) $\mathrm{S}_{9}$ and (d) $\mathrm{S}_{10}$

The proposed design also exhibits good current distribution in the radiating patch as illustrated in Figure 8 (a), (b), (c) and (d) respectively. The portion of the antenna which is excited at a particular frequency band is encircled in black.

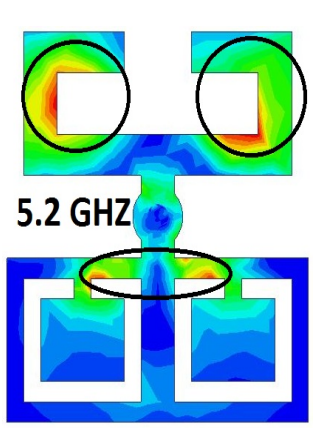

(a)

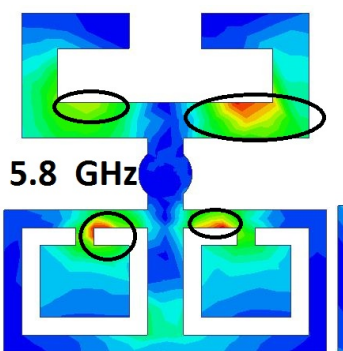

(c)

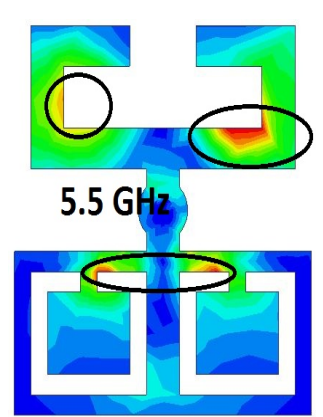

(b)
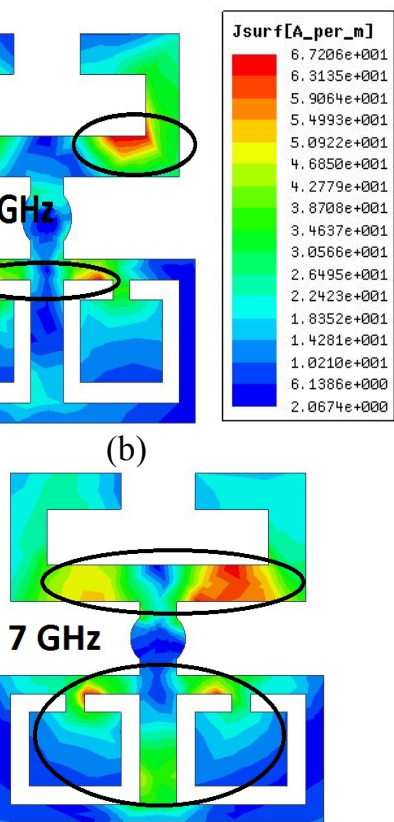

(d)

Figure 8. Current distribution of proposed design (a) 5.2, (b) 5.5, (c) 5.8 and (b) $7 \mathrm{GHz}$ 


\section{Results}

The proposed antenna design is simulated on ANSYS HFSS v.13.0 software and is fabricated using photolithographic process on RT Duroid 6006 substrate with dielectric constant $\left(\varepsilon_{r}\right)$, height $(\mathrm{h})$ and loss tangent $(\delta)$ of $6.15,2.54$ $\mathrm{mm}$ and 0.0019 , as illustrated Figure 9 (a). The set-up to measure the proposed antenna $S_{11}$ through VNA is depicted in Figure 9 (b). The antenna compared simulated and measured $S_{11}$ is further shown in Figure 9 (c).
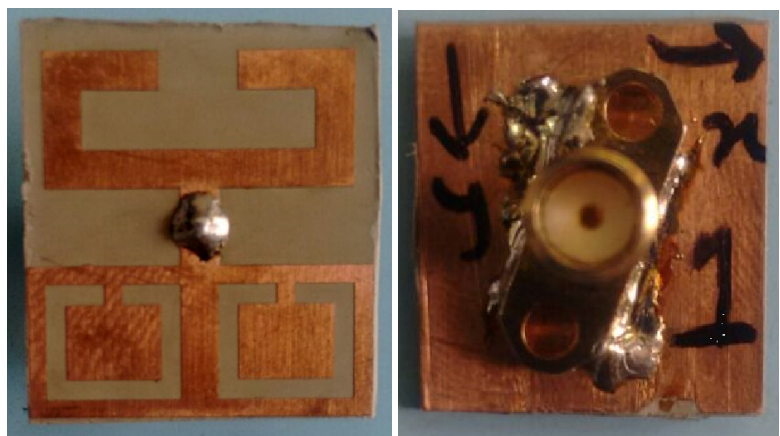

(a)

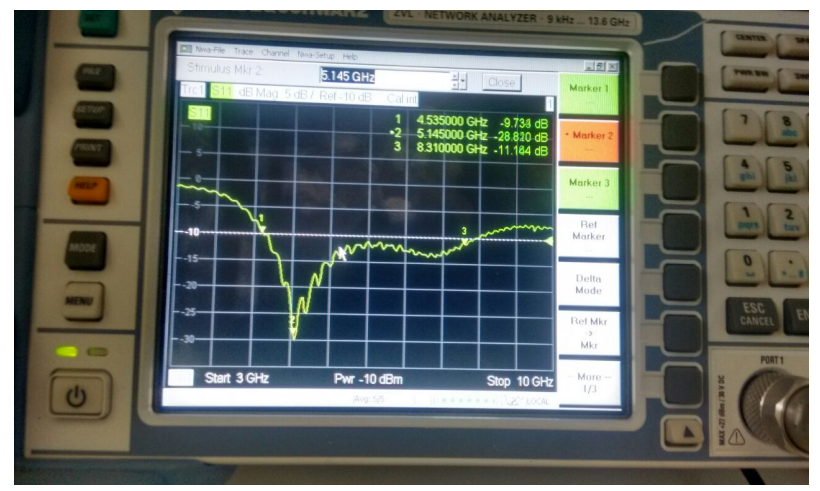

(b)

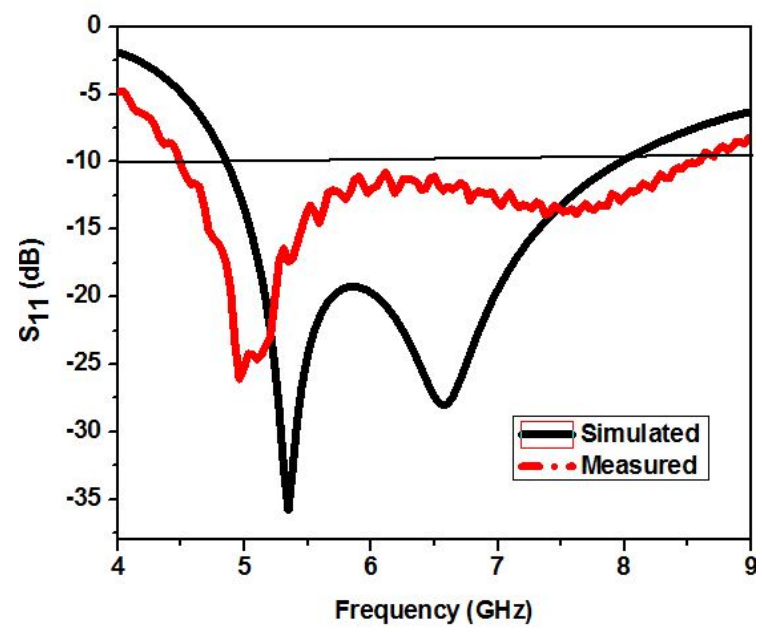

(c)

Figure 9. (a) Fabricated antenna front and back part (b) VNA set-up to measured $S_{11}$ (c) compared simulated and measured $\mathrm{S}_{11}$ of the proposed antenna.
It can be observed that the proposed structure has $S_{11}<$ $-10 \mathrm{~dB}$ bandwidth of about $3120 \mathrm{MHz}(4.85-7.97 \mathrm{GHz})$ in simulation and about $4060 \mathrm{MHz}(4.50-8.56 \mathrm{GHz})$ in measurement. The bandwidth enhancement can also be visualized by looking $\mathrm{S}_{11}$ results of the configuration "\#2" and the proposed configuration "\#4" from Figure 9. The slight glitches in the results may be due fabrication tolerance, soldering of SMA connector and manual error. However, the achieved impedance bandwidth is good and can easily meet the requirement of WLAN/WiMAX/Satellite applications.

The simulated and measured normalized radiation patterns of the antenna are illustrated in Fig. 10 and 11. For a patch antenna, the radiation pattern along $\mathrm{E}$ and H-plane has to be bi-directional and omnidirectional, respectively [16-17]. It can be observed from Fig. 10 and 11, that the proposed configuration has almost bi-directional and omnidirectional pattern in $\mathrm{E}$ and H-plane, respectively.

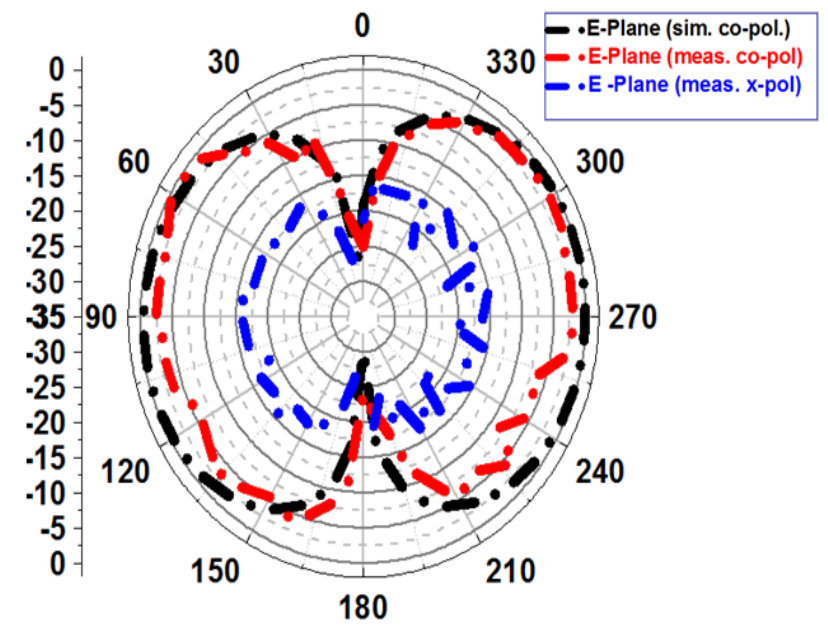

(a)

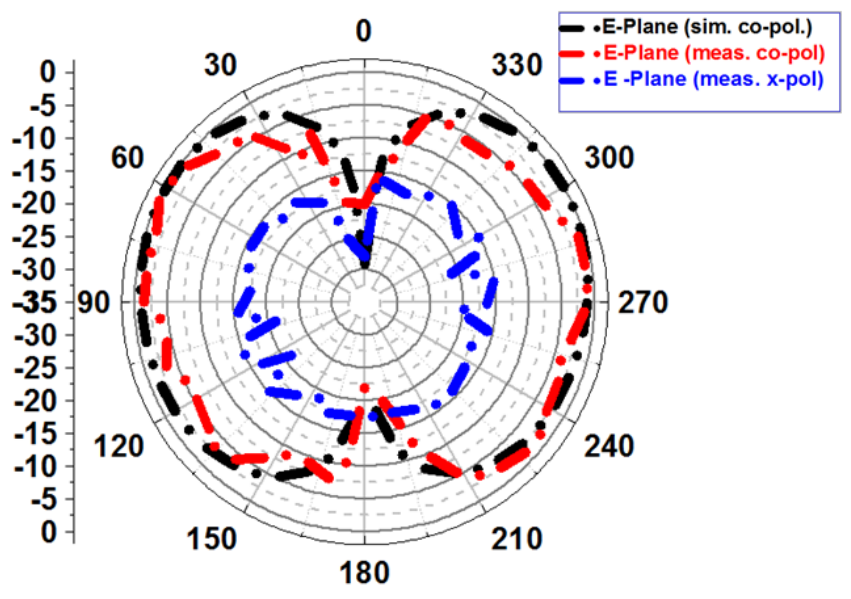

(b)

Figure 10. E- Plane normalized radiation pattern of the proposed design at (a) 5.3 and (b) $6.6 \mathrm{GHz}$ 


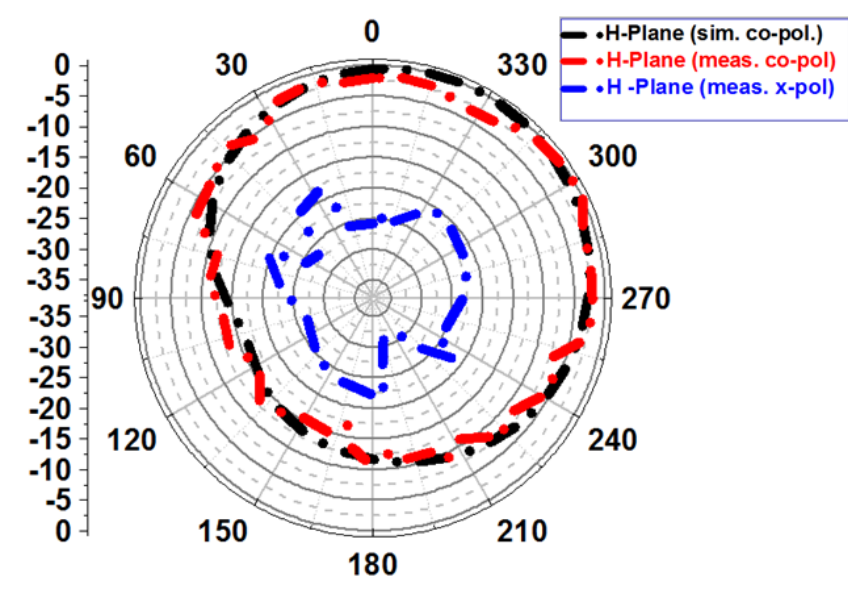

(a)

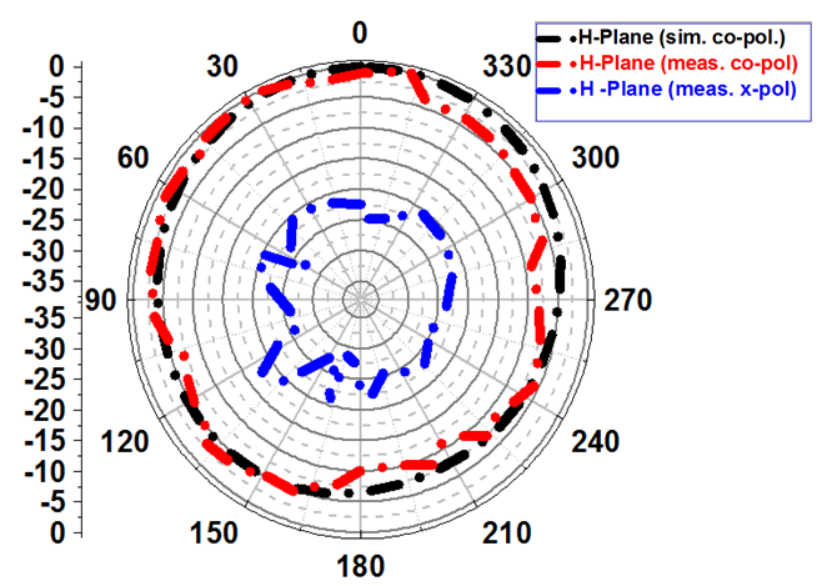

Figure 12. H- Plane normalized radiation pattern of the proposed design at (a) 5.3 and (b) $6.6 \mathrm{GHz}$

To know the significance of the designed antenna its compactness and bandwidth is compared with similar types of antennas present in the literature, and is depicted in Table 1. From the table it can be observed that the proposed structure is more compact and have greater bandwidth as compared to those studied in the literature.

Table 1. Comparision of the proposed design with those present in the literature.

\begin{tabular}{cccc}
\hline Ref. & $\begin{array}{l}\text { Dimensions } \\
\left(\mathrm{mm}^{3}\right)\end{array}$ & $\begin{array}{l}\text { Bandwidth } \\
\text { Range }(\mathrm{GHz})\end{array}$ & $\begin{array}{l}\text { Banwidth } \\
(\mathrm{MHz})\end{array}$ \\
\hline$[18]$ & $29 \times 29 \times 5.08$ & $1.19-1.25$ & 60 \\
{$[19]$} & $60 \times 50 \times 1.55$ & $2.6-5.1$ & 2500 \\
{$[20]$} & $30 \times 45 \times 1.6$ & $1.76-4.76$ & 3000 \\
{$[21]$} & $58 \times 54 \times 0.8$ & $1.8-3.6$ & 1800 \\
Prop. & $20 \times 18 \times 2.54$ & $4.85-7.97$ & 3120
\end{tabular}

\section{Conclusions}

A planar compact bandwidth enhanced antenna for WLAN/WiMAX/Satellite applications is presented. From the analysis it has been observed that the SRR is responsible for the resonance at $6 \mathrm{GHz}$, while bandwidth enhancement has been accomplished by two C-shaped slots. Parametric analysis shows that the slight variations in the optimized dimension degrade the antenna performance drastically. The omni-directional radiation pattern obtained in H-plane, favors the usage of the proposed antenna in remote applications and fading conditions. The antenna has a volume of only $20 \times 18 \times 2.54 \mathrm{~mm}^{3}$, and thus can easily be integrated with wireless end terminal devices. Compact size, good gain and stable radiation pattern makes the proposed design suitable for the aforementioned wireless applications.

\section{References}

[1] Antenna Theory, C.Balanis, Wiley, $2^{\text {nd }}$ edition (1997), Chapter 14.ISBN0-471-59268-4.

[2] R. K. Prasad, D. K. Srivastava and J. P. Saini, "Gain and bandwidth enhancement of rectangular microstrip antenna by loading slot," 2016 International Conference on Innovation and Challenges in Cyber Security (ICICCS-INBUSH), Noida, 2016, pp. 304-307.

[3] Islam, M. M., Islam, M. T., Samsuzzaman, M., \& Faruque, M. R. I. (2015). Compact metamaterial antenna for UWB applications. Electronics Letters, 51(16), 1222-1224..

[4] Ali. T., Mohammad Saadh, Biradar, R.C., J. Anguera and A. Andujar A miniaturized metamaterial slot antenna for wireless applications Int. J. Electron. Commun. (AEÜ) 82 (2017), 368-382.

[5] Ali, T., \& Biradar, R. (2017). A compact multiband antenna using $\lambda / 4$ rectangular stub loaded with metamaterial for IEEE $802.11 \mathrm{~N}$ and IEEE 802.16 E. Microwave and Optical Technology Letters, 59(5), 1000-1006..

[6] Ali, T., Saadh Aw, M., \& Biradar, R. (2018). A fractal quad-band antenna loaded with L-shaped slot and metamaterial for wireless applications. International Journal of Microwave and Wireless Technologies, 1-9. doi:10.1017/S1759078718000272

[7] Veselago, V. G. (1968). The electrodynamics of substances with simultaneously negative values of and

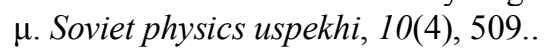

[8] A. S. Abbas and M. K. Abdelazeez, "Bandwidth enhancement of single notch planar UWB antenna with single step feed line," 2015 IEEE Jordan Conference on Applied Electrical Engineering and Computing Technologies (AEECT), Amman, 2015, pp. 1-6.

[9] Lizzi, L., Ferrero, F., Monin, P., Danchesi, C., \& Boudaud, S. (2016, July). Design of miniature antennas for IoT applications. In Communications and Electronics (ICCE), 2016 IEEE Sixth International Conference on (pp. 234-237). IEEE.

[10] Smith, D. R., et al. "Determination of effective permittivity and permeability of metamaterials from reflection and transmission coefficients." Physical Review B 65.19 (2002): 195104.

[11] A.M. Nicolson and G.F. Ross. Measurement of the intrinsic properties of materials by time- domain techniques. IEEE Trans Instrum Meas. 1970:19:377382 
[12] D. R. Smith, D. C. Vier, T. Koschny and C. M. Soukoulis. Electromagnetic parameter retrieval from inhomogeneous metamaterials. Phys. Rev. E, Stat. Phys. Plasmas Fluids Relat. Interdiscip. Top., vol. 71, Mar. 2005, Art. ID 036617.

[13] Szabo Z., Park G. H., Hedge R. \& Li E. P. A unique extraction of metamaterial parameters based on Kramers-Kronig relationship. IEEE Transactions on Microwave Theory and Techniques 2010:58(10):26462653.

[14] X. Chen, T. M. Grzegorczyk, B. I. Wu, J. Pacheco and J. A. Kong. Robust method to retrieve the constitutive effective parameters of metamaterials. Phys. Rev. E, Stat. Phys. Plasmas Fluids Relat. Interdiscip. Top., vol. 70, Feb. 2004, Art. ID 016608

[15] Szabo Z., Park G. H., Hedge R. \& Li E. P. A unique extraction of metamaterial parameters based on Kramers-Kronig relationship. IEEE Transactions on Microwave Theory and Techniques 2010:58(10):26462653.

[16] Ali, T., AW, M. S., Biradar, R. C., Andújar, A., \& Anguera, J. (2018). A miniaturized slotted ground structure UWB antenna for multiband applications. Microwave and Optical Technology Letters, 60(8), 2060-2068.

[17] Ali, T., \& Biradar, R. C. (2017). A Miniaturized Volkswagen Logo UWB Antenna with Slotted Ground Structure and Metamaterial for GPS, WiMAX and WLAN Applications. Progress In Electromagnetics Research C, 72, 29-41.

[18] Ali, T., Mohammad Saadh, A. W., Pathan, S., \& Biradar, R. C. A Miniaturized Circularly Polarized Coaxial Fed Superstrate Slot Antenna for L-band Application. Internet Technology Letters. doi.org/10.1002/it12.21.

[19] Li, Q., Feresidis, A. P., Mavridou, M., \& Hall, P. S. (2015). Miniaturized double-layer EBG structures for broadband mutual coupling reduction between UWB monopoles. IEEE Transactions on Antennas and Propagation, 63(3), 1168-1171.

[20] Othman, M. A., Abuelfadl, T. M., \& Safwat, A. M. (2013). Dual and wide-band inductively-loaded dipolebased antennas for WLAN/UMTS applications. IEEE Transactions on Antennas and Propagation, 61(3), 1430-1435.

[21] Kharche, S., Reddy, G. S., Gupta, R. K., \& Mukherjee, J. (2017). Wide band circularly polarised diversity antenna for satellite and mobile communication. IET Microwaves, Antennas \& Propagation, 11(13), 18611867. 